\title{
RETRACTED ARTICLE: Field observations of Weddell seal (Leptonychotes weddellii) twins at Terra Nova Bay in the Ross Sea, Antarctica
}

\author{
Hyunjun $\mathrm{Cho}^{1} \cdot$ Yejin $\mathrm{Kim}^{1,2} \cdot$ Won Young Lee ${ }^{1}$ (D)
}

Received: 24 May 2021 / Revised: 13 August 2021 / Accepted: 23 August 2021 / Published online: 28 August 2021

(c) The Author(s), under exclusive licence to Springer-Verlag GmbH Germany, part of Springer Nature 2021

The authors have retracted this article because they discovered after publication that the observer did not observe the birth moment of the seal pup twins as stated in the article. The authors also stated that the observations were done daily throughout the survey period; however, they have now discovered that the observations were done weekly although it was done daily at near twin observation period. As these factors directly influenced the conclusions of the study, the authors have agreed to retract the paper. All authors agree to this retraction.

The online version of this article contains the full text of the retracted article as Supplementary Information.

Supplementary Information The online version contains supplementary material available at https://doi.org/10.1007/s00300-021-02935-z.

Won Young Lee

wonyounglee@kopri.re.kr

1 Division of Polar Life Sciences, Korea Polar Research Institute, Incheon 21990, Republic of Korea

2 School of Earth and Environmental Sciences, Seoul National University, Seoul 08826, Republic of Korea 